\title{
Pneumoencephalocele and CSF leak as complications of the congenital temporal bone malformation
}

\author{
Emilia Karchier ${ }^{*}$, Kazimierz Niemczyk ${ }^{1}$, Rober Bartoszewicz ${ }^{1}$ and Kateryna Terentieva ${ }^{2}$ \\ ${ }^{1}$ Department of Otolaryngology, Medical University of Warsaw, Poland \\ ${ }^{2}$ Department of Otorhinolaryngology, Ukrainian National State Medical University, Ukraine
}

\begin{abstract}
Introduction: Pneumocephalus is a rare complication in otology associated with the presence of the air in the cranial cavity. It may be a result of erosion of the skull base due to a neoplasm, infection, neurosurgical procedures, trauma, otogenic infections, mastoid surgeries and idiopathic causes. The cerebrospinal fluid leaksotorrhea may be congenital, traumatic, iatrogenic, or spontaneous. The management of a CSF leak varies by etiology as well.

Case report: A twenty-seven-year-old man with a history of occipital craniotomies in 2005 and 2006 for removal of astrocytoma of the cerebellum, was admitted due to pneumoencephalocele lasting for two years. He underwent an endoscopic transsphenoidal reconstruction of the skull base of the middle fossa and frontal craniotomy for anterior fossa reconstruction in the other hospital. These procedures did not lead to an improvement, but resulted in complications, i.e. meningitis, swallowing disorders and, consequently, pneumonia. From this time, the patient has been in poor physical condition unable to work. A surgery of the reconstruction of the skull base of the middle fossa, successively on the right and the left side, separated by several months was performed. During the procedure, a congenital defect of the temporal bone was found in the form of a dehiscence of the middle cranial fossa, allowing the air flow from the middle ear to the internal auditory canal and further to the skull cavity. The skull base was sealed with pieces of muscle, fascia and tissue glue. After the surgery, the symptoms gradually improved and hearing was preserved. A follow-up MRI was performed and there was no remnant air in the intracranial cavity.

Conclusion: A congenital malformation of the temporal bone can cause spontaneous cerebrospinal fluid (CSF) leaks and pneumocephalus. The closure of air cells or fistula from the area of the inner ear is an effective method of treating and preventing pneumocephalus and otorrhea resulting from congenital malformations of the temporal bone.
\end{abstract}

\section{Introduction}

Intracranial presence of air may occur post-traumatically, postoperatively, especially after neurosurgical and otosurgical procedures, as a complication of otitis media, a consequence of the tumour of the skull base and very rarely- spontaneously in case of anatomical variants of the temporal bone if the air cells are connected with the space of the cranial cavity $[1,2]$. The first case of pneumocephalus was reported by Chiari in 1884, during an autopsy done in patient with ethmoiditis [3]. In 1926 Dandy reported the first case of otogenic pneumocephalus [4]. Otorrhea may also occur posttraumatically, iatrogenically and spontaneously, when diagnosis is especially difficult because the symptoms may be subtle, transient or recurrent, as otitis media with effusion, hearing loss or meningitis. In such cases a patient requires an extremely detailed diagnostic process. In children the source of otorrhea is usually a cochlear capsule: in oval window, the facial nerve canal or structures of the inner ear [5]. There are several dozen cases of meningitis associated with the congenital inner ear disorder described in the literature [6]. The mean age of onset of meningitis was 3.6 years (0.1-14 years) and the average time of diagnosis of the congenital malformation of the skull base (from the first episode of meningitis) was 3.44 years $(0.00-10.00$ years). In the reported cases of otorrhea, the most common symptom was unilateral hearing loss in $70 \%$ of patients, whereas $10 \%$ of patients had normal hearing [7]. In the literature there were also described two fatal cases of meningitis due to a congenital malformation of the inner ear. In 1933 Kline described the case of a 54-year-old man with right-sided otitis media with effusion for several days who died due to meningitis.
The autopsy revealed a fistula in the temporal bone [8]. The case of a 44-year-old man, with fluids and hearing deterioration for 2 months was described in 1982 by Neely [9]. Intraoperatively, dehiscences of tegmen were diagnosed. The authors reported also the head injury in the patient one year before the onset of the disease. Recently, in 2017, Martinez-Perez showed a case of pneumocephalus in a 75-year-old woman after the implantation of a ventriculoperitoneal shunt 15 years earlier [10]. The patient was consulted 2 months earlier due to coma and disturbances of consciousness. The CT scan showed a bone defect in the skull base of the middle cranial fossa. Temporal craniotomy was performed to close the dura and temporal bone defect

\section{Case report}

A twenty-seven-year-old man was admitted to the Department of Otolaryngology of Medical University of Warsaw with a two-year history of the presence of the air in the cranial cavity (Figures 1 and 2 ). The patient was physically rehabilitated with full verbal and logical contact. He had a history of occipital craniotomies for removal of

${ }^{\star}$ Correspondence to: Emilia Karchier, Department of Otolaryngology, Medical University of Warsaw, Zwirki i Wigury 61, 02-091 Warszawa, Poland, Tel: +48 22 57209 13, E-mail: ekarchier@wum.edu.pl

Key words: pneumocephalus, MRI, temporal bone, endoscopic transsphenoidal reconstruction

Received: July 06, 2018; Accepted: July 22, 2018; Published: July 25, 2018 


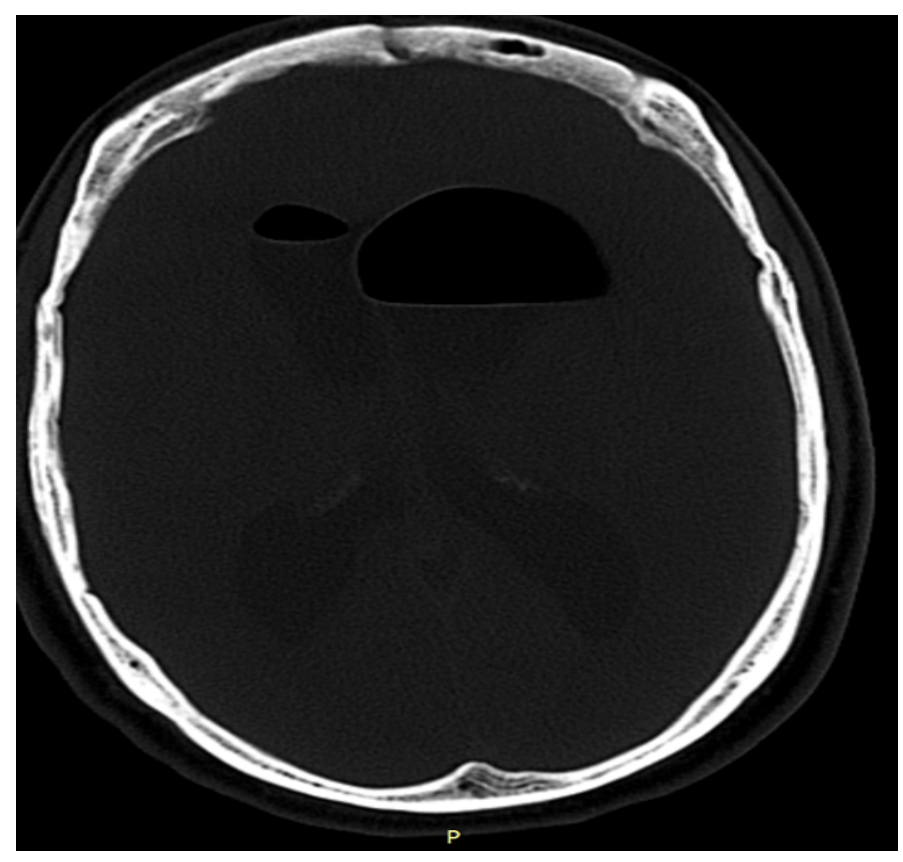

Figure 1. CT scan of the head pneumoencephalocele

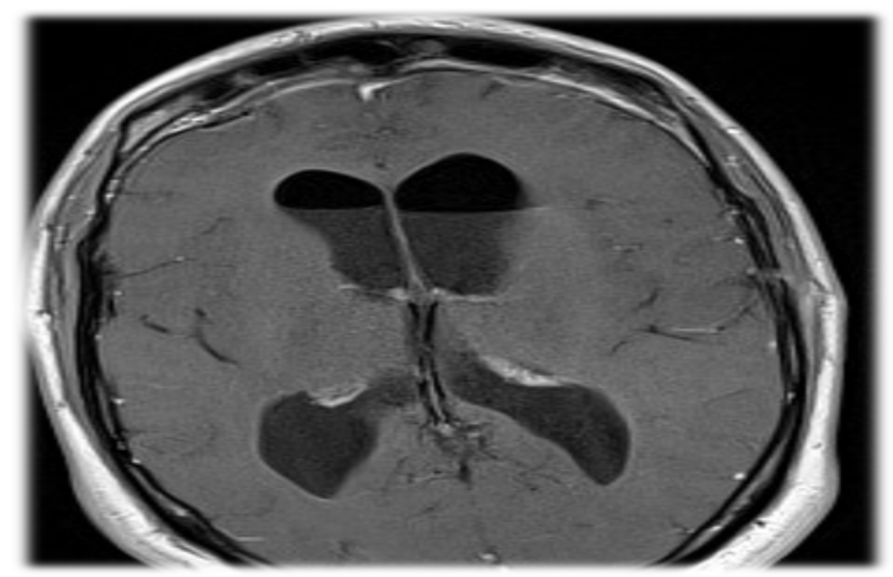

Figure 2. MRI of the brain pneumoencephalocele

astrocytoma of the cerebellum in the 15-th and the 16-th year of life (Figure 3). Since that time, he had right vocal cord paralysis. He had viral meningitis in the 7-th year of life.

The onset of the disease occurred in 2015 during the patient's stay in Great Britain. The patient so far in good health condition, suffered severe headaches during exertion at work. He was admitted to the Nottingham University Hospital in Great Britain with headache and vomiting for three weeks. MRI and CT scan reported the presence of the air in the ventricles, posterior fossa, CPA and near the petrous carotid. A surgery was recommended, and the patient underwent transsphenoidal decompression and repair of sphenoid sinus defect. Nasal CSF leak appeared postoperatively and unfortunately, the patient developed meningitis for which long course of antibiotics was administered. Control CT scan showed recurrent pneumocephalus. Due to nasal CSF leak the patient was operated again (transsphenoidal fat and fascia pack and lumbar drain of CSF). Because of the inefficiency he was subjected to another surgery of reconstruction of the skull base in the area of the sphenoid sinus and afterwards to frontal craniotomy for reconstruction of the anterior cranial fossa. Since the procedure, the patient experienced dysfunction of speech and difficulties in swallowing liquids. The patient was treated for three episodes of aspiration pneumonia with tazocin and clarithromycin. From April 2016 to May 2017 the patient was nourished via gastrostomy. Since this time, the patient has been in poor physical condition unable to work.

In our department a surgery of the middle cranial fossa on the right side was performed in April 2017. During the procedure, a defect of the temporal bone was found in the form of the dehiscences and fistulas from the internal auditory canal to the cranial cavity and deficit of the dura, allowing the air to flow from the middle ear to the skull cavity. The skull base was sealed with the pieces of muscle, fascia and tissue glue. After the surgery on the right side, the symptoms gradually improved and hearing was preserved, but the presence of the air in the left lateral ventricle was still confirmed (Figures 4 and 5). Therefore, a

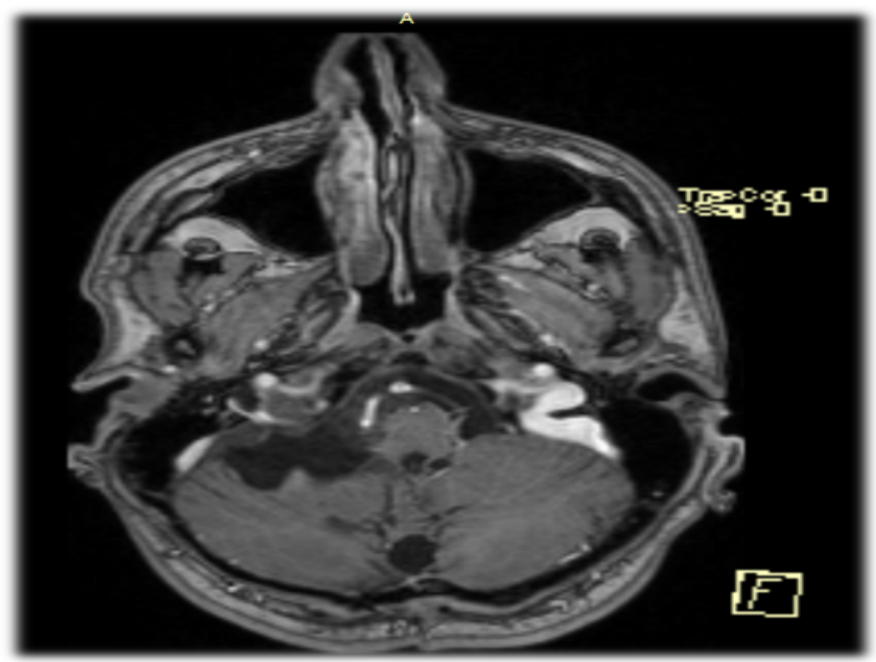

Figure 3. MRI of the brain, the picture after the removal of astrocytoma by occipital craniotomy a few years ago

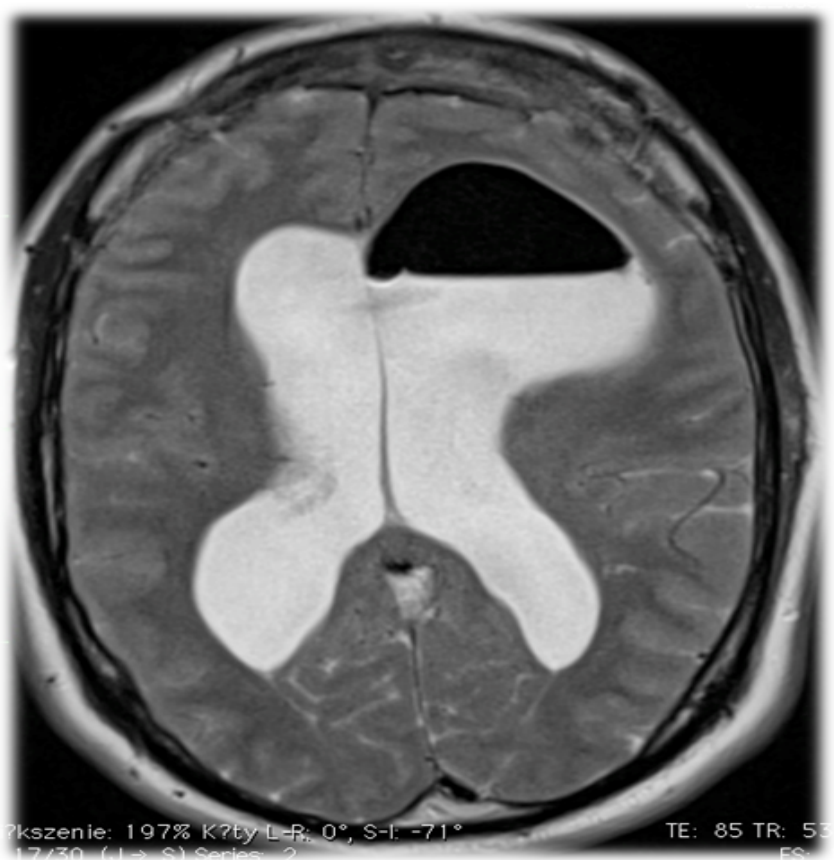

Figure 4. MRI of the brain after reconstruction of the skull base through middle fossa approach on the right side - the presence of the air in the left lateral ventricle 
similar procedure on the other side was performed after four months, obtaining a complete resolution (Figure 6). The postoperative period proceeded without complications. The patient was discharged from the hospital in satisfactory condition. A follow-up MRI was performed and it revealed no remnant air in the intracranial cavity (Figure 7). Finally, augmentation of the right vocal cord with hyaluronic acid was conducted to improve the voice.

\section{Discussion}

A bilateral congenital defect of the temporal bone roof is an extremely rare cause of otorrhea and the presence of air in the cranial cavity. In the studied patient not only a defect of skull base structure was detected, but also thinned, not fully functional dura mater in the area of air leak from the mastoid cells to the cranial cavity. It is supposed that a valve mechanism was formed in the patient during physical exertion that let the air into the cranial cavity, replacing the leaking cerebrospinal fluid that flew to the mastoid cell system, tympanic cavity

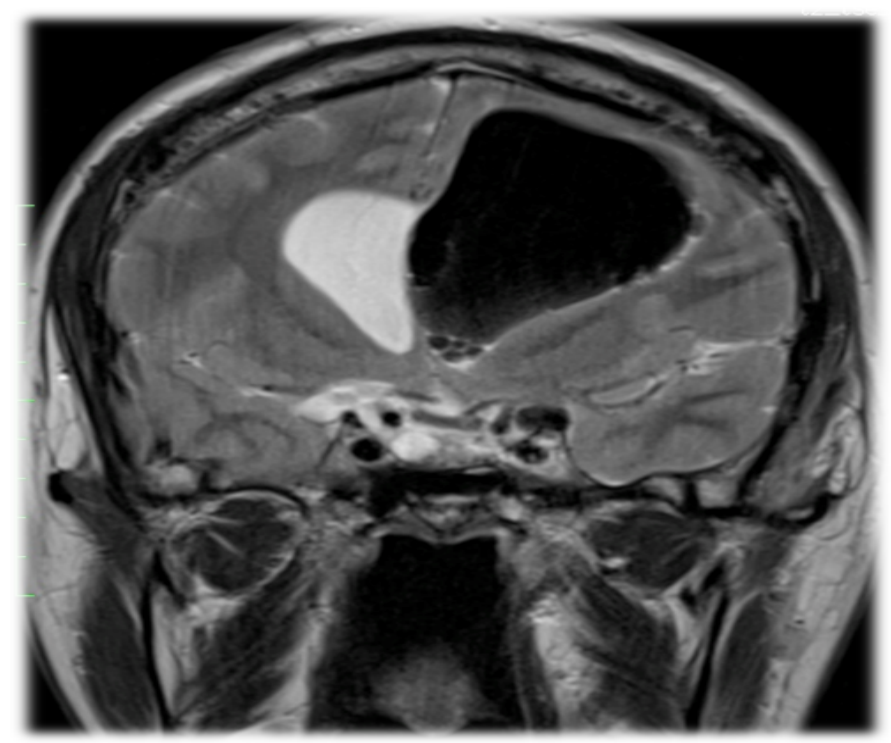

Figure 5. MRI of the brain after reconstruction of the skull base through middle fossa approach on the right side - the presence of the air in the left lateral ventricle

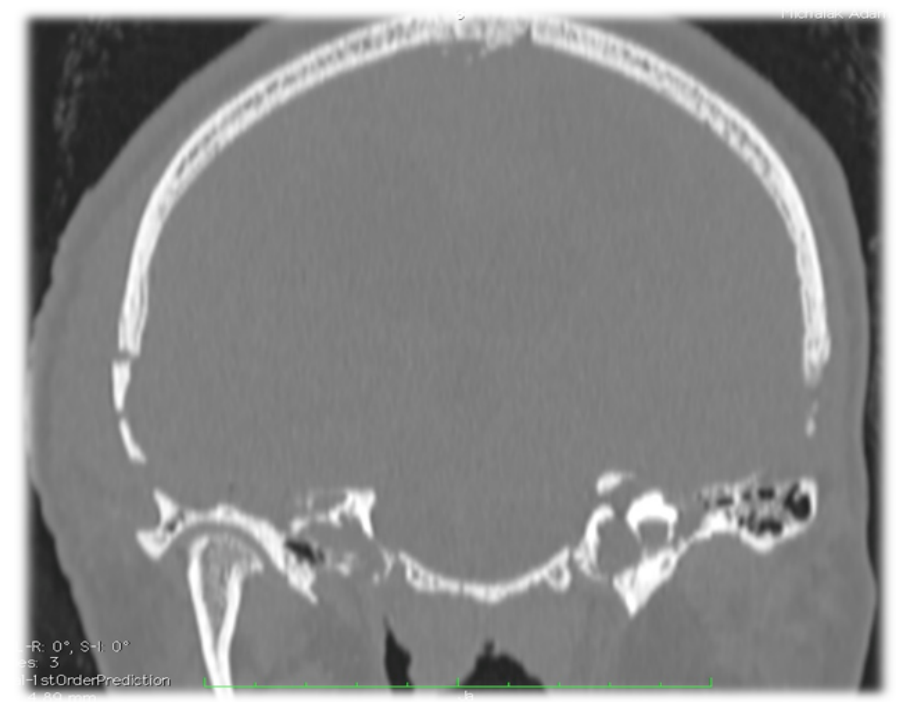

Figure 6. CT of the head two weeks after the second reconstruction of the skull base through middle fossa approach on the left side

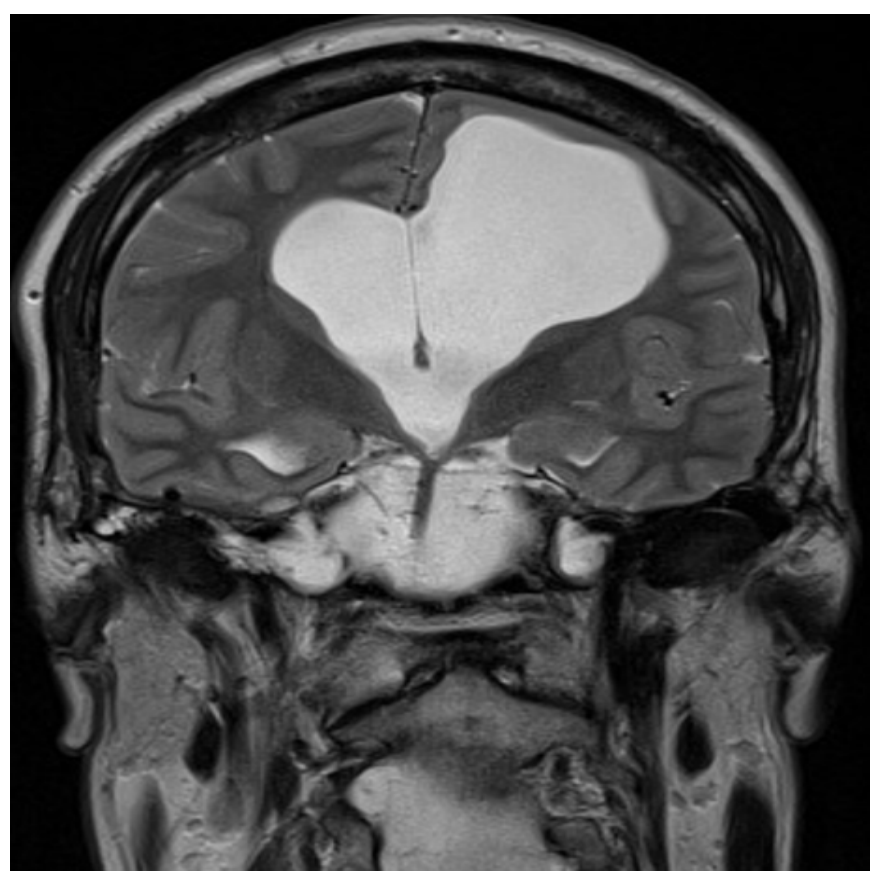

Figure 7. MRI of the brain after six months from the second reconstruction of the skull base through middle fossa approach on the left side

and further, through the Eustachian tube to the nasopharynx. The patient was previously subjected to numerous sealing procedures of the middle and anterior cranial fossa, but they appeared to be ineffective, which encouraged us to search for another location of a cavity in the skull base. Observing similar cases in the subject literature and based on the results of diagnostic imaging examinations of the patient, it was decided to perform a surgery through the middle cranial fossa approach in order to seal the roof of the temporal bone. After the intraoperative detection of fistulas enabling the communication between the internal auditory canal and the middle cranial fossa, a sealing material was used - temporal muscle fascia, temporal muscle and tissue glue in order to seal the minor fistulas in the temporal bone. Improvement on the operated side proved the effectiveness of the applied treatment, so it was decided to perform the same procedure on the left side. The treatment turned out to be effective, so the patient returned to the normal everyday activities. It can be hypothesized that the meningitis that the patient underwent at the age of 7 could have been associated with the present temporal bone structure defect as well.

\section{Conclusion}

A congenital defect of the temporal bone may be the cause of spontaneous otorrhea and pneumoencephalocele. The closure of the dehiscence of mastoid air cells or fistula from the inner ear area and internal auditory canal by access through the middle cranial fossa is an effective method of treatment and prevention of pneumocephalus and otorrhea resulting from a congenital defect of the temporal bone.

\section{References}

1. Markham JW (1967) The clinical features of pneumocephalus based upon a survey of 284 cases with report of 11 additional cases. Acta Neurochir (Wien) 16: 1-78. [Crossref]

2. Ferguson BJ, Wilkins RH, Hudson W, Farmer J Jr (1986) Spontaneous CSF otorrhea from tegmen and posterior fossa defects. Laryngoscope 96: 635-644. [Crossref]

3. Chiari $\mathrm{H}$ (1884) About a case of air accumulation in the ventricles of the human brain. Jir Heilkunde. 5: 383-391.

4. Dandy WE (1926) Pneumocephalus (intracranial pneumatocele or aerocele). Arch Surg 12: $949-982$. 
5. Schultz P, Stool S (1970) Recurrent meningitis due to a congenital fistula through stapes footplate. Am J Dis Child 120: 553-558. [Crossref]

6. Guindi GM (1981) Congenital labyrintho-tympanic fistula-a recently recognized entity in children. J Otolaryngol 10: 67-71. [Crossref]

7. Muzzi E, Battelino S, Gregori M, Pellegrin A, Orzan E (2015) Life-threatening unilateral hearing impairments. Review of the literature on the association between inner ear malformations and meningitis. Int J Ped Otorhinolaryngol 79: 1969-1974. [Crossref]
8. Kline OR (1933) Spontaneous cerebrospinal otorrhea. Arch Otolaryngol 18: 34-39.

9. Martinez-Perez R, Gomez E, Rayo N (2017) Spontaneous Tension Pneumocephalus : A Rare Complication of Shunting. World Neurosurg 100: 710.e11-710.e13. [Crossref]

10. Rao N, Redleaf M (2016) Spontaneous Middle Cranial Fossa Cerebrospinal Fluid Otorrhea in Adults. Laryngoscope 126: 464-468. [Crossref]

Copyright: $(02018$ Karchier E. This is an open-access article distributed under the terms of the Creative Commons Attribution License, which permits unrestricted use, distribution, and reproduction in any medium, provided the original author and source are credited. 